\title{
Optimization, Modelling and Analysis of Air-Cooled Battery Thermal Management System for Electric Vehicles
}

Muhammad Muddasar*

U.S.-Pakistan Centre for Advanced Studies in Energy, NUST, Islamabad, 44000

*Corresponding Author

\section{ABSTRACT}

Electric Vehicles (EVs) are the need of the hour due to growing climate change problems linked with the transportation sector. Battery Thermal Management System (BTMS), which is accountable for certifying safety and performance of lithium-ion batteries (LiB), is the most vital part of an EV. LiB has auspicious gravimetric energy density but the heat generation due to chemical reactions inside a LiB during charging and discharging causes temperature rise which has a direct effect on LiB performance and safety. This study specifically focuses on aircooled BTMS, defines different types of air-cooled BTMS (active and Passive), discusses limitations associated with air-cooled BTMS, and investigates different optimization techniques and parameters to improve performance of air-cooled BTMS. Maintaining temperature within optimum range and uniform temperature distribution between cells of a battery pack are the major design parameters for improving the performance and efficiency of air-cooled BTMS. Various optimization techniques including cell arrangement with a battery pack, air-flow channel optimization, and air inlet/outlet position variations are discussed and each technique is thoroughly reviewed. Finally, it's noted that passive air-cooled BTMS is not that effective for long-distance vehicles so most researchers shifted their focus toward active air-cooled BTMS. Active air-cooled BTMS requires a lot of power for effective performance. Lastly, the most recent field of air-cooled BTMS technology which is Air-Hybrid BTMS is discussed and declared a very promising solution for overcoming major limitations associated with air-cooled BTMS. 


\section{CONTENTS}

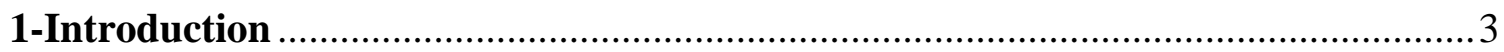

2-Battery Thermal Management System …….......................................................

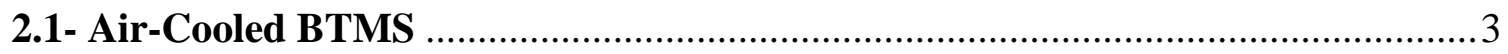

3- Air-Cooled BTMS- Optimization Techniques ….............................................

3.1-Cell Arrangement - Battery Layout Optimization ...........................................

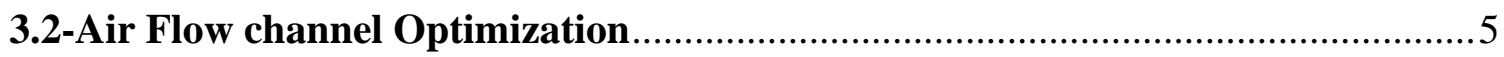

3.3- Duct Inlet/ Outlet Position or Fan Placement ............................................... 6

4- Advantages \& Challenges of Air-Cooled BTMS ................................................ 8

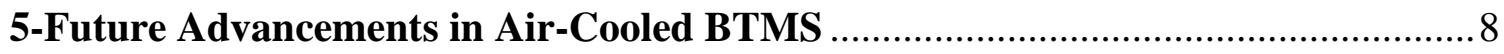

6-References 


\section{1-Introduction}

The growing climate change concerns can be addressed by implementing sustainable development goal (SDG) 13. With the aim to accomplish this goal, extensive sustainable transport optimization have to be pursued. This can be done by replacing conventional fossil fuel powered vehicles with electric vehicles (EVs) [1]. Most suitable energy storage device for EVs are lithium-ion batteries (LiBs) [2], however, the most critical parameter that effects performance, safety and life of LiBs is temperature [3]. Variation in environmental conditions and internal heat release by chemical reactions occur during the operating cycle of LiB causes temperature change which is usually inevitable and poses serious performance and safety problems. [4] Battery thermal management systems (BTMS) is the best solution to mitigate that temperature change issue in LiBs [5].

\section{2-Battery Thermal Management System}

BTMS is a complex system configured for EVs to heat battery pack (BP) if temperature is less than lower temperature limit and to cool BP if temperature is above upper limit [6]. Operating temperature range for $\mathrm{LiBs}$ is suggested to be kept between $15^{\circ} \mathrm{C}$ and $30^{\circ} \mathrm{C}$ and temperature difference between two adjacent modules should be below $5^{\circ} \mathrm{C}$ to avoid performance losses in LiBs [7]. BTMS should perform cooling and heating to maintain temperature, provide insulation to prevent temperature change and have vents to remove hazardous gases [8]. Researchers have identified many BTMS technologies depending on working fluid medium for cooling but the scope of this review research proposal is air-cooled BTMS because the temperature of battery rises due to internal chemical reactions is most critical problem in LiBs performance.

\section{1- Air-Cooled BTMS}

Air cooling BTMS is the most traditional strategy used to perform BTMS functions by utilizing external air with or without preconditioning. Both natural convection which is also known as passive cooling and forced convection known as active cooling can be used as air-cooled BTMS strategies are used commercially [9]. This review focuses on optimization techniques for air-cooled BTMS like modification in battery layout, air-flow channel layout modification and, in case of forced convection, cooling fan placement [10]. 


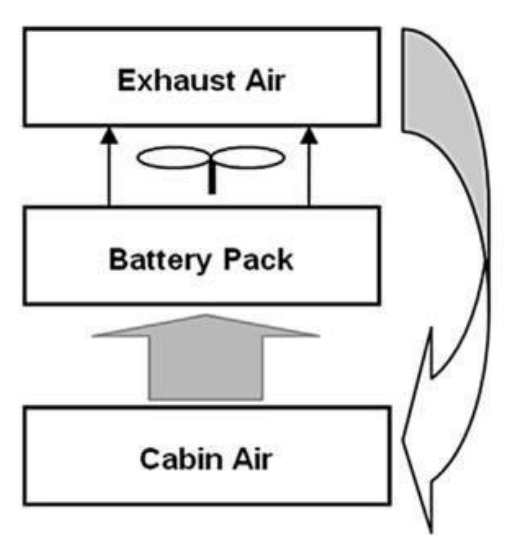

(a)

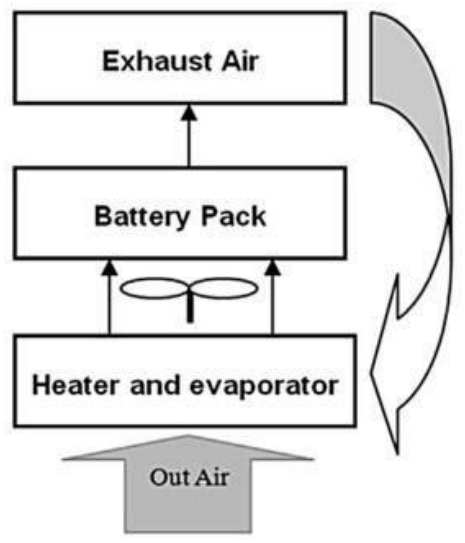

(b)

Schematic of thermal management using air (a) passive heating and cooling using cabin air (b) active heating and cooling using outside air [19].

\section{3- Air-Cooled BTMS- Optimization Techniques}

There have been many studies performed on air-cooled BTMS in recent years to improve cooling performance as air has low heat capacity and thermal conductivity. Many researchers only set air temperature at appropriate room temperature that's why pre-conditioned air can be considered as a medium for battery cooling [11]. This section includes detailed study of each optimization technique.

\section{1-Cell Arrangement - Battery Layout Optimization}

Major design variable to improve cooling performance of an air-cooled BTMS is the arrangement of cells inside a BP of LiB. Yang et al. (2015) developed a thermal model to investigate thermal performance of the cylindrical battery packs $(6 \times 10)$ of LiFePO4. The results of longitudinal and traverse spacing for both aligned and staggered cell arrangements are discussed. The average temperature rise of individual cells increase as longitudinal spacing of staggered arrangement increase but decrease for aligned cell arrangement. In case of transverse spacing, average temperature rise increase as spacing increase for both aligned and staggered alignment but with improved temperature uniformity. Most suitable results are achieved for aligned arrangement with optimized air inlet duct width with longitudinal and transverse spacing to be $34 \mathrm{~mm}$ and $32 \mathrm{~mm}$ respectively with maximum temperature to be 33.9 and temperature difference of 0.93 [12].

Fan et al. (2019) performed experimental analysis for cooling performance of a forced aircooled BTMS with aligned, staggered and cross 32 cylindrical lithium ion cells arrangement. 
Results revealed that aligned battery pack has least maximum temperature difference with best cooling effectiveness for $2 \mathrm{C}$ rate. They also found that cooling effectiveness increase with increases in air velocity but it decreases overall BTMS efficiency as power consumption is directly proportional to air velocity [13].

Kang et al. (2020) investigated forced air-cooled BTMS for two different arrangement methods for 16 cells which are square and rectangular arrangement. Thermal analysis was performed on both arrangements. They concluded that for same number of cells, battery pack can have different temperature characteristics and internal temperature distribution varies as shape of pack changes from square to rectangle [14].

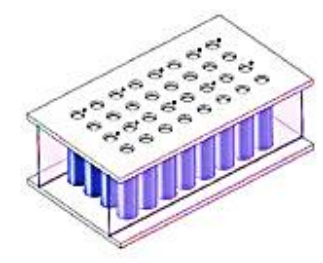

Aligned battery pack

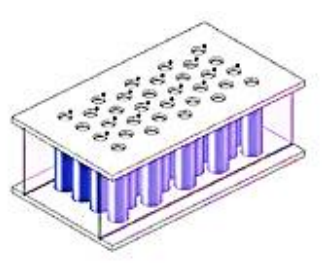

Staggered battery pack

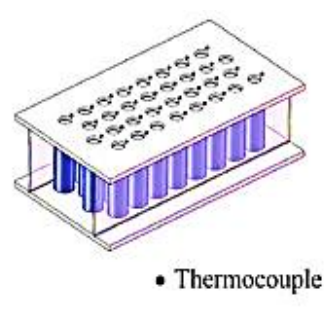

Cross battery pack

(a)

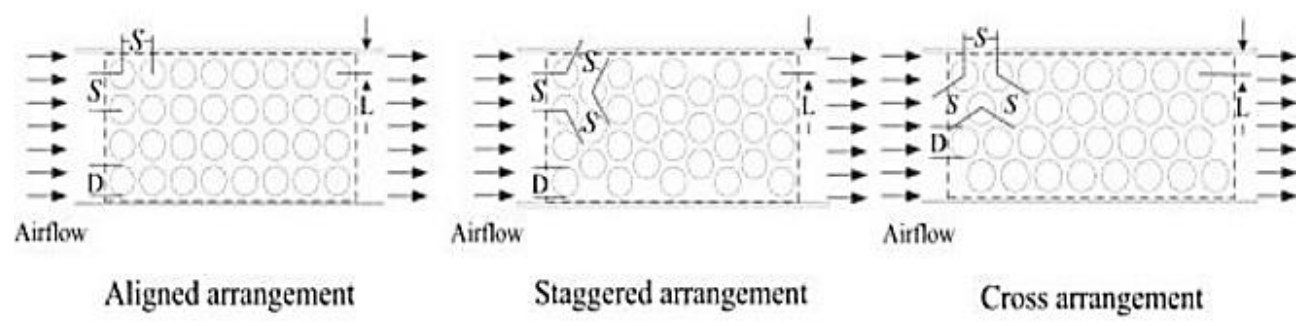

(b)

(a) Schematic diagram of aligned, staggered and cross-arranged battery packs;

(b) Structural projection of three arranged battery packs. [14][23]

\section{2-Air Flow channel Optimization}

This techniques focuses on improving BTMS cooling performance by optimizing air flow channel in BP as airflow channels have direct relation with cell temperature and temperature uniformity. Na et al. (2018) examined 2 air flow configurations for 10 cylindrical cells. They compared 2 configurations which are Unidirectional Air Flow (UDAF) and Reversed Layered Air Flow (RDAF) and concluded that RLFD significantly reduce temperature difference amongst cells and greatly improve temperature consistency. The result shows that average temperature reduction for RDAF is reduced by $46 \%$ [15]. 
Chen et al. (2018) developed a flow resistance numerical model for forced air-cooled BTMS with $U$ type flow to calculate air flow rates in channel. Nested loop procedure with Newton method was used for optimizing angle of plenums and the width air inlet and outlet ducts. Comparing optimized and initial U-type flow indicated that after optimization $70 \%$ reduction in cell temperature difference and $32 \%$ reduction in power consumption by fans. They also indicated that width of inlet and outlet air ducts were the main design factors to optimize [16]. Sun et al. (2014) developed a "Z-type" air flow channel by applying Design of Experiments method (DoE method) for pouch type LiB. They investigated that variations of air flow rates of cooling channels can be significantly reduced by using tapered inlet and outlet [17].

Liu et al. (2019) presented an innovative "J-Type" air flow configuration by mixing up Z and U type air flow configurations. A surrogate-based optimization was performed for BTMS having $\mathrm{U}, \mathrm{Z}$ and $\mathrm{J}$ type air flow configurations. They depicted that $35.3 \%, 46.6 \%$ and $31.1 \%$ reduction in temperature rise of cells under $\mathrm{U}, \mathrm{Z}$ and $\mathrm{J}$ type configuration respectively. The temperature uniformity can be enhanced for J-type configuration by switching working mode between U-type and Z-type alternatively by controlling opening degree of valves [18].

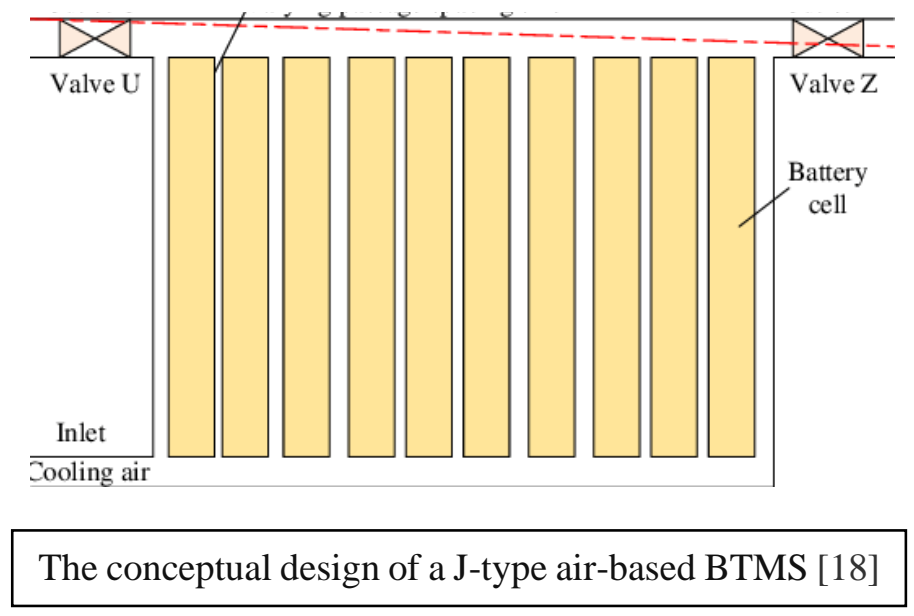

\section{3- Duct Inlet/ Outlet Position or Fan Placement}

Minimizing fan power consumption in forced-air cooled BTMS can be optimized by examining the best location to place inlet and outlet air vents so that minimum power is required for circulation of air in air flow channels. Wang et al. (2015) suggested the ideal temperature range for forced-air cool BTMS is about from $20^{\circ} \mathrm{C}$ to $35^{\circ} \mathrm{C}$. They also discovered that when fan position is on top of module, the highest temperature occurs at the outmost cells as air speed along edges was slow. They also revealed that forced-air cool BTMS is not required for areas with ambient temperature below $20^{\circ} \mathrm{C}$ but if high discharge rates are required than forced-air cool BTMS was suggested [20]. 
Jiaqiang et al. (2018) instigated thermal characteristics of the 60 cylindrical cells module by varying the relative positions of inlet and outlet air vents. They developed a CFD model to perform experiment. They examined that air vents on different sides had better cooling performance than air vents on top and bottom or on the same side of module. Lastly, they presented a unique design which had baffles plates within the module to enhance air circulation [21].

Chen at al. (2020) examined the effect of asymmetrical air flow in 5 BTMS configurations numerically and confirmed results by physical experiments. They also developed symmetrical air flow models by optimizing inlet and outlet air vents and suggested that symmetrical air flow models had better cooling performance. Maximum temperature difference between cells for symmetrical air flow models was less than $3.0 \mathrm{~K}$. They also revealed that symmetrical air flow models had maximum cell temperature difference reduced by at least $43 \%$ and energy consumption by at least $33 \%$ [22].

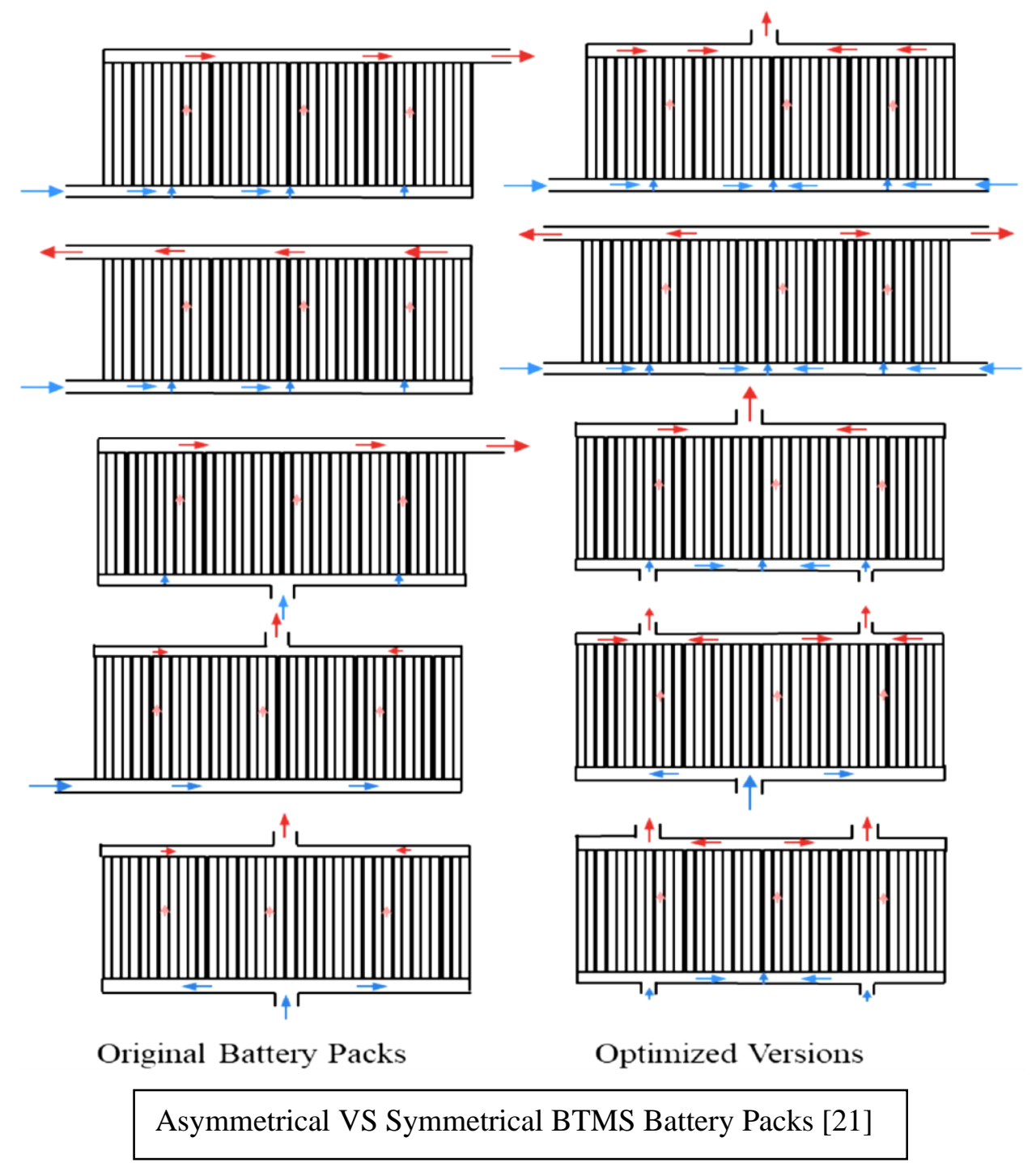




\section{4- Advantages \& Challenges of Air-Cooled BTMS}

Air cooled BTMS has advantages like:

> Simple construction

$>$ Safety and reliability in its operation

$>$ Ease of maintenance and implementation

Main challenge for air-cooled BTMS is the low specific heat capacity and low heat transfer coefficient of air as compared liquid cooling medium. This causes decrease in cooling performance for this BTMS when using it for highly dense battery module because dense battery module have high capacity and suitable for long-distance EVs but also high heat generation and less spacing between cells.

Passive Air-cooled BTMS, which is suitable for low capacity battery modules, have very less capability for cooling dense battery module which results in problems like thermal runaway and passenger comfort issues . Optimization can be done for air-cooled BTMS for dense battery modules by introducing forced air and increasing volume of air or preconditioning of air but these techniques require excess power which is not suitable [23].

\section{5-Future Advancements in Air-Cooled BTMS}

In order to prevent loss in cooling performance faced by air-cooled BTMS while using it for long-distance EVs, researches should focus in areas for integrating air-cooled BTMS with other cooling medium to develop "Hybrid BTMS" which benefits from low cost, compactness and easy implementation of air-cooled BTMS and good heat transfer coefficient (convective) of other cooling medium like water or PCM.

The Air-Hybrid Battery Thermal Management System provides opportunity to overcome a major limitation which is air low heat transfer coefficient. Novel water spray evaporation is integrated in an air-cooled BTMS by Yang et al. (2019) [24] and PCM integrated with aircooled BTMS by Qin et al. (2019) [25] are some recent advancements in this field.

The aim of this review is to make aware future researchers with the past important work done in the field of air-cooled BTMS and help them to make innovative air cooled BTMS by improving past works. 


\section{6-References}

1. UNEP. (2015). Analysis of the transport relevance of each of the 17 SDGs.

2. S. M. A. S. Bukhari, J. Maqsood, M. Q. Baig, S. Ashraf and T. A. Khan, "Comparison of Characteristics -- Lead Acid, Nickel Based, Lead Crystal and Lithium Based Batteries," 2015 17th UKSim-AMSS International Conference on Modelling and Simulation (UKSim), Cambridge, 2015, pp. 444-450.

3. T.M. Bandhauer, S. Garimella, T.F. Fuller, A critical review of thermal issues in lithium-ion batteries, Electrochem. Soc. 158 (2011) R1-R25.

4. K. Smith, C.Y. Wang, Power and thermal characterization of a lithium-ion battery pack for hybrid-electric vehicles, Power Sources 160 (2006) 662-673.

5. Wang, Q., Jiang, B., Li, B., \& Yan, Y. (2016). A critical review of thermal management models and solutions of lithium-ion batteries for the development of pure electric vehicles. Renewable and Sustainable Energy Reviews, 64, 106-128.

6. Jim William Dunham, E. E. (2015). United States Patent No. US10573940B2.

7. Pesaran AA., Santhanagopalan S, Kim GH. Addressing the impact of temperature extremes on large format li-ion batteries for vehicle applications (presentation). No. NREL/PR-5400-58145. National Renewable Energy Lab. (NREL), Golden, CO (United States), 2013.

8. Pesaran AA. Battery thermal management in EVs and HEVs: issues and solutions. Nevada: Adv. Automot. Batter. Conf. Las Vegas; 2001.

9. Liu, H., Wei, Z., He, W., \& Zhao, J. (2017). Thermal issues about Li-ion batteries and recent progress in battery thermal management systems: A review. Energy Conversion and Management, 150, 304-330.

10. Lu, M., Zhang, X., Ji, J., Xu, X., \& Zhang, Y. (2020). Research progress on power battery cooling technology for electric vehicles. Journal of Energy Storage, 27, 101155.

11. Kim, Jaewan \& Oh, Jinwoo \& Lee, Hoseong. (2018). Review on Battery Thermal Management System for Electric Vehicles. Applied Thermal Engineering. 149. 10.1016/j.applthermaleng.2018.12.020. 
12. Yang, N., Zhang, X., Li, G., \& Hua, D. (2015). Assessment of the forced air-cooling performance for cylindrical lithium-ion battery packs: A comparative analysis between aligned and staggered cell arrangements. Applied Thermal Engineering, 80, 55-65.

13. Fan, Y., Bao, Y., Ling, C., Chu, Y., Tan, X., \& Yang, S. (2019). Experimental study on the thermal management performance of air cooling for high energy density cylindrical lithium-ion batteries. Applied Thermal Engineering.

14. Kang, Deokhun \& Lee, Pyeong-Yeon \& Yoo, Kisoo \& Kim, Jonghoon. (2020). Internal thermal network model-based inner temperature distribution of high-power lithium-ion battery packs with different shapes for thermal management. Journal of Energy Storage. 27.

15. Na, X., Kang, H., Wang, T., \& Wang, Y. (2018). Reverse layered air flow for Li-ion battery thermal management. Applied Thermal Engineering, 143, 257-262.

16. Chen, K., Song, M., Wei, W., \& Wang, S. (2018). Structure optimization of parallel air-cooled battery thermal management system with U-type flow for cooling efficiency improvement. Energy, 145, 603-613.

17. Sun, H., \& Dixon, R. (2014). Development of cooling strategy for an air cooled lithiumion battery pack. Journal of Power Sources, 272, 404-414.

18. Liu, Y., \& Zhang, J. (2019). Design a J-type air-based battery thermal management system through surrogate-based optimization. Applied Energy, 252, 113426.

19. Bajaj, H. \& Kini, Chandrakant. (2019). Thermal management systems for EV's and HEV's. ARPN Journal of Engineering and Applied Sciences. 14. 265-269.

20. Wang, T., Tseng, K. J., \& Zhao, J. (2015). Development of efficient air-cooling strategies for lithium-ion battery module based on empirical heat source model. Applied Thermal Engineering, 90, 521-529.

21. Jiaqiang, E., Yue, M., Chen, J., Zhu, H., Deng, Y., Zhu, Y., ... Kang, S. (2018). Effects of the different air cooling strategies on cooling performance of a lithium-ion battery module with baffle. Applied Thermal Engineering.

22. Chen, K., Chen, Y., She, Y., Song, M., Wang, S., \& Chen, L. (2019). Construction of effective symmetrical air-cooled system for battery thermal management. Applied Thermal Engineering, 114679.

23. Lu, M., Zhang, X., Ji, J., Xu, X., \& Zhang, Y. (2020). Research progress on power battery cooling technology for electric vehicles. Journal of Energy Storage, 27, 101155. 
24.Yang, Y., Yang, L., Du, X., \& Yang, Y. (2019). Pre-cooling of air by water spray evaporation to improve thermal performance of lithium battery pack. Applied Thermal Engineering, 114401.

25.Jilte, R. D., Kumar, R., Ahmadi, M. H., \& Chen, L. (2019). Battery thermal management system employing phase change material with cell-to-cell air cooling. Applied Thermal Engineering, 114199. 